\title{
ON SOME NONUNIFORM CASES OF THE WEIGHTED SOBOLEV AND POINCARÉ INEQUALITIES
}

\author{
F. I. MAMEDOV AND R. A. AMANOV
}

\begin{abstract}
Weighted inequalities $\|f\|_{q, \nu, B_{0}} \leq C \sum_{j=1}^{n}\left\|f_{x j}\right\|_{p, \omega_{j}, B_{0}}$ of Sobolev type (supp $\left.f \subset B_{0}\right)$ and of Poincaré type $\left(\bar{f}_{\nu, B_{0}}=0\right)$ are studied, with different weight functions for each partial derivative $f_{x_{j}}$, for parallelepipeds $B_{0} \subset E_{n}, n \geq 1$. Also, weighted inequalities $\|f\|_{q, \nu} \leq C\|X f\|_{p, \omega}$ of the same type are considered for vector fields $X=\left\{X_{j}\right\}, j=1, \ldots, m$, with infinitely differentiable coefficients satisfying the Hörmander condition.
\end{abstract}

\section{§1. INTRODUCTION}

This paper is devoted to weighted versions of the classical inequalities

$$
\|f\|_{q, B_{0}} \leq C\|\nabla f\|_{p, B_{0}} \quad\left(q=\frac{p n}{n-p}, n>p \geq 1\right)
$$

of Sobolev type if supp $f \subset B_{0}$ and of Poincaré type if $\bar{f}_{B_{0}}=0$ (see [1). In terms of a certain system of parallelepipeds (balls of the quasimetric $|\cdot|_{\bar{\sigma}}$ ), we study the inequalities

$$
\|f\|_{q, \nu, B_{0}} \leq C \sum_{j=1}^{n}\left\|f_{x_{j}}\right\|_{p, \omega_{j}, B_{0}} \quad(q \geq p \geq 1), \quad f \in \operatorname{Lip}\left(B_{0}\right),
$$

of the above types, with different weight functions for each partial derivative $f_{x_{j}}$ (Theorems 2.1 and 2.2). For $p=2$, such inequalities may turn out to be useful when applying the general method (see [2, 3, 4]) for the study of regularity, the Harnack inequality, and the Wiener criterion for weak solutions of degenerate elliptic equations of the form

$$
\frac{\partial}{\partial x_{i}}\left(a_{i j}(x) \frac{\partial u}{\partial x_{j}}\right)=0
$$

where $A=\left\|a_{i j}(x)\right\|$ is a real symmetric matrix such that there exists $\mu \in(0,1]$ with

$$
\mu \sum_{j=1}^{n} \omega_{j}(x) \xi_{j}^{2} \leq A \xi \cdot \xi \leq \mu^{-1} \sum_{j=1}^{n} \omega_{j}(x) \xi_{j}^{2}
$$

for all $\xi \in E_{n}$.

This case has been studied relatively poorly, in comparison with the uniform case $\left(\omega_{j}(x) \equiv \omega(x), j=1,2, \ldots, n\right)$; a summary of the results pertaining to the uniform case was given, e.g., in [5, Theorem 5].

2000 Mathematics Subject Classification. Primary 46 E35.

Key words and phrases. Sobolev and Poincaré inequalities, Carnot-Carathéodory metric, Besicovitch property.

The work of the first author was supported in part by INTAS (grant no. 8792). 
A modern approach to the study of these issues is related to applying the CarnotCarathéodory metric in the case of the degenerate vector field

$$
X_{1}=\omega_{1}(x) \frac{\partial}{\partial x_{1}}, \quad \ldots, \quad X_{n}=\omega_{n}(x) \frac{\partial}{\partial x_{n}},
$$

which, in its turn, is given in terms of some family of curves ("horizontal curves"). Since the coefficients in (1.5) may fail to be smooth, in [3, 8, 9] the Hörmander condition was replaced with appropriate conditions of a geometric nature.

We also consider the weighted inequalities

$$
\|f\|_{q, \nu, B_{0}} \leq C\|X f\|_{p, \omega, B_{0}} \quad(q \geq p \geq 1), \quad u \in \operatorname{Lip}\left(B_{0}\right),
$$

for vector fields $X=\left\{X_{j}\right\}, j=1, \ldots, m$, with $C^{\infty}$-coefficients that satisfy the Hörmander condition [10] of finite rank

$$
\operatorname{rank} \operatorname{Lie}\left[X_{1}, \ldots, X_{m}\right]=n,
$$

where $B_{0}$ is a metric ball of a special Carnot-Carathéodory metric determined by the vector field.

Inequalities (1.6) find applications to the study of regularity, the Harnack inequality, etc., for solutions of subelliptic equations

$$
\sum_{j=1}^{m} X_{j}^{*}(A(x, u, X u))=0
$$

(see, e.g., [11, 12, 13]), where $X_{j}^{*}$ denotes the formal conjugate to $X_{j}$, and $A: D \times E_{1} \times$ $E_{n} \rightarrow E_{n}$ is a Carathéodory function satisfying the coercivity condition (see [13]).

\section{§2. Definitions, notation, And formulation of Results}

To adequately formulate our results, we list some definitions and notation. The $n$ dimensional Euclidean space of points $x=\left(x_{1}, \ldots, x_{n}\right), n \geq 1$, is denoted by $E_{n}$. Suppose some metric (or quasimetric) $\rho(x, y)$ is given in $E_{n}$. We put $\rho(x)=\rho(x, 0)$. In particular, given a system $\bar{\sigma}=\left\{\sigma_{1}, \sigma_{2}, \ldots, \sigma_{n}\right\}$ of positive numbers, we can consider the quasimetric $\rho(x, y)=\max _{1 \leq i \leq n}\left\{\left|x_{i}-y_{i}\right|^{1 / \sigma_{i}}\right\}$; by analogy with the Euclidean metric, we denote this $\rho(x, y)$ by $|x-y|_{\bar{\sigma}} . B(x, r)=\left\{y \in E_{n}: \rho(x, y)<r\right\}$ is the metric ball centered at $x \in E_{n}$ of radius $r>0 ; d(\Omega)=\sup \{\rho(x, y): x, y \in \Omega\}$ is the metric diameter of a domain $\Omega$. We write $r(B)$ for the radius of a metric ball $B$. We put $e_{j}(B)=\sup \left\{\left|x_{j}-y_{j}\right|: x, y \in B\right\}$; this is the length of the $j$ th edge of $B$. For a measurable set $E$, its Lebesgue measure is denoted by $|E|$. For an integrable nonnegative function $f$ and a measurable set $E$, we denote $f(E)=\int_{E} f(x) d x$.

$\operatorname{Lip}(D)$ is the space of functions Lip-continuous in $D . \operatorname{Lip}_{0}(D)$ will denote the subspace in $\operatorname{Lip}(D)$ formed by the functions that vanish on the boundary $\partial D$ of the domain $D \subset E_{n}$.

We denote by $L_{p, \nu}(D)$ the space of measurable functions in $D$ for which the norm

$$
\|f\|_{p, \nu, D}=\left(\int_{D}|f(x)|^{p} \nu(x) d x\right)^{1 / p}, \quad p \geq 1,
$$

is finite. If this leads to no confusion, we shall simply write $\|f\|_{p, \nu}$ in place of $\|f\|_{p, \nu, D}$. The quantities

$$
\bar{f}_{\nu, D}=\frac{1}{\nu(D)} \int_{D} f(x) \nu(x) d x, \quad \bar{f}_{D}=\bar{f}_{1, D},
$$

are the mean values of $f$ in $D$. 
Let $\omega$ be a nonnegative locally integrable function, $\Omega \Subset E_{n}$ a compact set, and $\rho$ a metric (or quasimetric) in $E_{n}$. We say that $\omega$ satisfies the condition $A_{p}\left(=A_{p}(\Omega, \rho, d x)\right)$, $1 \leq p<\infty$, if

$$
\begin{gathered}
\left(\frac{1}{|B|} \int_{B} \omega(x) d x\right)\left(\frac{1}{|B|} \int_{B} \omega^{-1 /(p-1)}(x) d x\right)^{p-1} \leq C_{p}<\infty \quad \text { for } 1<p<\infty, \\
\frac{1}{|B|} \int_{B} \omega(x) d x \leq C_{1} \underset{x \in B}{\operatorname{ess} \inf } \omega(x) \quad \text { for } \quad p=1
\end{gathered}
$$

for all metric balls $B=B(x, t)$ with $x \in \Omega, 0<t \leq 5 d(\Omega)$. We say that a function $v$ satisfies the condition $A_{\infty}\left(=A_{\infty}(\Omega, \rho, d x)\right)$ if there exist constants $C, \delta>0$ such that

$$
v(E) / v(B) \leq C(|E| /|B|)^{\delta}
$$

for any metric ball $B=B(x, t)$ with $x \in \Omega, 0<t \leq 5 d(\Omega)$, and any subset $E \subset B$.

Suppose we are given a pair of nonnegative functions $v, \omega$ such that $v, \omega^{-1 /(p-1)} \in$ $L_{1, \text { loc }}$ for $1<p<\infty$ and $\omega^{-1} \in L_{\infty}$,loc for $p=1$ (these conditions will be assumed throughout).

We say that the functions $v, \omega$ satisfy the local balance condition in $\Omega$ if

$$
\frac{r(B)}{r\left(B_{1}\right)} \frac{\left|B_{1}\right|}{|B|}\left(\frac{v(B)}{v\left(B_{1}\right)}\right)^{1 / q}\left(\frac{\omega^{-1 /(p-1)}(B)}{\omega^{-1 /(p-1)}\left(B_{1}\right)}\right)^{(p-1) / p} \leq C_{p q}<\infty
$$

for all metric balls $B=B(x, t), B_{1}=B(x, 2 d(\Omega))$, where $x \in \Omega, 0<t \leq 2 d(\Omega)$.

If $\omega$ satisfies $A_{p}$, then (2.4) turns into the condition

$$
\frac{r(B)}{r\left(B_{1}\right)} \cdot\left(\frac{v(B)}{v\left(B_{1}\right)}\right)^{1 / q} \leq C_{p q}^{\prime}\left(\frac{\omega(B)}{\omega\left(B_{1}\right)}\right)^{1 / p}
$$

In Theorem 2.2, for the quasimetric $|\cdot|_{\bar{\sigma}}$ and a fixed ball $\Omega=B_{0}$, instead of $A_{\infty}$ we assume the following condition $A_{\infty}^{\prime}\left(=A_{\infty}\left(\Omega,|\cdot|_{\bar{\sigma}}, \chi_{\Omega} d x\right)\right)$ : there exist $C, \delta>0$ such that

$$
\frac{v(E \cap \Omega)}{v(B \cap \Omega)} \leq C\left(\frac{|E \cap \Omega|}{|B \cap \Omega|}\right)^{\delta}
$$

for any metric ball $B=B(x, t), x \in \Omega, 0<t \leq 5 d(\Omega)$ and any subset $E \subset B$.

The Sobolev inequality to be proved in the case of the quasimetric $|\cdot|_{\bar{\sigma}}$ looks like the following.

Theorem 2.1. Let $1 \leq p \leq q<\infty$, and let $B_{0}$ be a fixed ball of the quasimetric $|\cdot|_{\bar{\sigma}}$ in $E_{n}$. Suppose that $v \in A_{\infty}\left(B_{0},|\cdot|_{\bar{\sigma}}, d x\right)$. If the condition

$$
|B|^{-1} e_{j}(B)(v(B))^{1 / q}\left(\omega_{j}^{-1 /(p-1)}(B)\right)^{(p-1) / p} \leq A_{p q}<\infty, \quad j=1, \ldots, n,
$$

is fulfilled for any metric ball $B=B(x, t), x \in B_{0}, 0 \leq t \leq 2 d\left(B_{0}\right)$, then there exists a positive number $C_{0}$, depending on $n, q$ and the constants $C$ and $\delta$ occurring in (2.3), such that for any $f \in \operatorname{Lip}_{0}\left(B_{0}\right)$ we have

$$
\left(\int_{B_{0}}|f(x)|^{q} v(x) d x\right)^{1 / q} \leq C_{0} A_{p q} \sum_{j=1}^{n}\left(\int_{B_{0}}\left|f_{x}\right|^{p} \omega_{j}(x) d x\right)^{1 / p} .
$$

Remark 2.1. The proof shows that, instead of the quasimetric $|\cdot|_{\bar{\sigma}}$, in Theorem 2.1 (as well as in Theorem 2.2) we could choose an arbitrary quasimetric with the Besicovitch property, provided that the metric balls are convex (we need this for obtaining estimate $(3.7))$.

The next result concerns the Poincaré inequality for the quasimetric $|\cdot|_{\bar{\sigma}}$. 
Theorem 2.2. Let $1 \leq p \leq q<\infty$, and let $B_{0}$ be a fixed ball of the quasimetric $|\cdot|_{\bar{\sigma}}$ in $E_{n}$. Suppose that $v$ is a function satisfying (2.6). If the condition

$$
\begin{array}{r}
e_{j}(B)|B|^{-1}\left(v\left(B \cap B_{0}\right)\right)^{1 / q}\left(\omega_{j}^{-1 /(p-1)}\left(B \cap B_{0}\right)\right)^{(p-1) / p} \leq A_{p q}<\infty, \\
j=1, \ldots, n,
\end{array}
$$

is fulfilled for any quasimetric ball $B=B(x, t), x \in B_{0}, 0<t \leq 2 d\left(B_{0}\right)$, then there exists a positive number $C_{0}$, depending on $n, q$ and the constants $C$ and $\delta$ occurring in (2.6), such that for any $f \in \operatorname{Lip}\left(B_{0}\right)$ we have

$$
\left(\int_{B_{0}}\left|f-\bar{f}_{v, B_{0}}\right|^{q} v(x) d x\right)^{1 / q} \leq C_{0} A_{p q} \sum_{j=1}^{n}\left(\int_{B_{0}}\left|f_{x_{j}}\right|^{p} \omega_{j}(x) d x\right)^{1 / p} .
$$

As applications of Theorems 2.1 and 2.2, we present the following two examples, which pertain to the case where $p=2$.

Example 2.1. Let $\beta=\left(\beta_{1}, \ldots, \beta_{n}\right)$ be a fixed vector with nonnegative components, and let $\eta \geq 0$ be such that $\max _{1 \leq j \leq n} \beta_{j} \leq\left(\sum_{k=1}^{n} \beta_{k}+n \eta\right) / 2$. Next, let the numbers $\sigma_{i}$ for the quasimetric $|\cdot|_{\bar{\sigma}}$ be defind by $\sigma_{i}=\frac{\eta+\beta_{i}}{2}, i=1, \ldots, n$. Suppose that

$$
\frac{1}{q}-\frac{1}{2}+\frac{\eta}{n \eta+\sum_{k=1}^{n} \beta_{k}}=0 .
$$

Then there exists a positive constant $C=C(n, \eta, \beta)$ such that for any $f \in \operatorname{Lip}_{0}\left(B_{R}^{a}\right)$ we have

$$
\left(\frac{1}{\left|B_{R}^{a}\right|} \int_{B_{R}^{a}}|f(x)|^{q} d x\right)^{1 / q} \leq C_{0} R^{\eta / 2} \sum_{j=1}^{n}\left(\frac{1}{\left|B_{R}^{a}\right|} \int_{B_{R}^{a}}|x|_{\bar{\sigma}}^{\beta_{j}}\left|f_{x_{j}}\right|^{2} d x\right)^{1 / 2} .
$$

Example 2.2. Let vectors $\beta=\left(\beta_{1}, \ldots, \beta_{n}\right), \bar{\sigma}=\left(\sigma_{1}, \ldots, \sigma_{n}\right)$ and numbers $q, \eta$ be as in Example 2.1. Then there exists a constant $C=C(n, \eta, \beta)$ such that for any $f \in \operatorname{Lip}\left(B_{R}^{a}\right)$ we have inequality (2.12), where $f$ is replaced with $f-\bar{f}_{B_{R}^{a}}$.

Remark 2.2. It is of interest to consider the approach where the parallelepipeds of the metric $|\cdot|_{\bar{\sigma}}$ are replaced by an arbitrary system of parallelepipeds $\{P\}$, without any reference to a metric or quasimetric; however, $\{P\}$ and the system of weights $\left\{v, \omega_{1}, \ldots, \omega_{n}\right\}$ must be coordinated so as to allow the verification of (2.7) (or (2.9)). Also, $\{P\}$ should possess the following properties.

1) The Besicovitch property: let $A \subset E_{n}$ be an arbitrary bounded set (in the Euclidean metric), and let $G \subset\{P\}$ be a subsystem that covers $A$, i. e., there exists $\{P(x)\} \subset G$ such that $A \subset \bigcup_{x \in A} P(x)$. Then the covering $G$ admits a finite or countable subcovering $\left\{P_{i}\right\}_{i \in N}$ of finite multiplicity $C_{n}>0$, i.e.,

$$
\exists\left\{P_{i}\right\}_{i \in N} \subset G, \quad A \subset \bigcup_{i \in N} P_{i}, \quad \sum_{i \in N} \chi_{p_{i}}(x) \leq C_{n} .
$$

2) For every $\gamma \in\left(0, d_{0}\right)$, where $d_{0} \in(0,1)$ is some number, every compact set $K \subset E_{n}$, and almost every $x \in \operatorname{int} K$, there exists a parallelepiped $P_{\gamma}(x) \in G$ such that

$$
\left|P_{\gamma}(x) \backslash K\right|=\gamma\left|P_{\gamma}(x)\right| .
$$

Note that a certain criterion for a system of parallelepipeds in $E_{n}$ to possess the Besicovitch property was proved in 14 . 
The next results of the paper pertain to weighted inequalities of Sobolev type (supp $f \subset$ $\left.B_{0}\right)$ and of Poincaré type $\left(\bar{f}_{\nu, B_{0}}=0\right)$ :

$$
\begin{aligned}
\left(\frac{1}{v\left(B_{0}\right)} \int_{B_{0}}|f|^{q} v(x) d x\right)^{1 / q} & \leq C r\left(B_{0}\right)\left(\frac{1}{\omega\left(B_{0}\right)} \int_{B_{0}}|X f|^{p} \omega(x) d x\right)^{1 / p} \\
q & \geq p \geq 1, \quad f \in \operatorname{Lip}\left(B_{0}\right),
\end{aligned}
$$

for vector fields $X=\left\{X_{j}\right\}, j=1, \ldots, m$, where $X_{j}=\sum_{k=1}^{n} b_{j k}(x) \frac{\partial}{\partial x_{k}}$ with $C^{\infty}$-coefficients; we assume the Hörmander condition (1.7). For $f \in \operatorname{Lip}\left(E_{n}\right)$, we define $X_{j} f=$ $\sum_{k=1}^{n} b_{j k}(x) \frac{\partial f}{\partial x_{k}}, j=1,2, \ldots, m$; then $X f$ is the vector with the components $X_{j} f$ and with length $|X f|^{2}=\sum_{i=1}^{m}\left|X_{i} f\right|^{2}$.

Following the conventional definitions (see, e.g., 15, 16), we say that an absolutely continuous curve $\gamma:[a, b] \rightarrow E_{n}$ is admissible ("horizontal") if there exist functions $c_{j}(t)$, $a \leq t \leq b$, satisfying

$$
\sum_{j=1}^{n} c_{j}^{2}(t) \leq 1, \quad \dot{\gamma}=\sum_{j=1}^{n} c_{j}(t) X_{j}(\gamma(t))
$$

Let a distance $\rho(x, y)$ between $x, y \in E_{n}$ be defined as the infimum of all $T>0$ for which there exists an admissible curve $\gamma:[0, T] \rightarrow E_{n}$ with $\gamma(0)=x, y(T)=y$. The resulting space $\left(E_{n}, \rho\right)$ is metric; in the literature it is called the Carnot-Carathéodory space (for a modern presentation, see [7]). In [17, many important properties of the above metric can be found; in particular, we mention the doubling property for the Lebesgue measure of metric balls: for any compact set $\Omega \subset E_{n}$ there exists a number $C_{1} \geq 1$ such that

$$
\left|B\left(x_{0}, 2 r\right)\right| \leq C_{1}\left|B\left(x_{0}, r\right)\right|, \quad x_{0} \in \Omega,
$$

$0<r \leq 5 d(\Omega)$. The quantity $Q=\log _{2} C_{1}$ is called the size of homogeneity of the field relative to the compact set $K$. This quantity plays the role of a dimension $(n)$ for vector fields with property (1.7) (see, e.g., [18, 19]). Inequality (2.17) implies that

$$
\left|B\left(x_{0}, \rho\right)\right| /\left|B\left(x_{0}, r\right)\right| \geq C(\rho / r)^{Q}, \quad 0 \leq \rho<r,
$$

where $0<r \leq 5 d(\Omega)$.

The space $\left(E_{n}, \rho\right)$ described above is locally compact and equipped with a curve length. In [18, Lemma 3.7], with the help of the Arzelà lemma, it was proved that there exists a continuous curve $\gamma_{x y}(t)$ that connects two given points $x, y \in E_{n}$ and is such that for any $z \in\left\{\gamma_{x y}(t)\right\}$ we have

$$
\rho(x, y)=\rho(x, z)+\rho(z, y) .
$$

The Poincaré inequality for vector fields was obtained in 20 for $q=p>1$. This result was refined in 21 to cover the case where $1<p<Q, q=p Q /(Q-p)$. The important case where $p=1, q=Q /(Q-1)$ was proved in 22]; this case merges with (more precisely, is equivalent to) the isoperimetric inequality

$$
\min (|E \cap B|,|B \backslash E|)^{(Q-1) / Q} \leq C\left(\frac{r(B)}{|B|^{1 / Q}}\right) P_{X}(E ; B),
$$

where $C>0$ depends on $n, Q$, the compact set $\Omega$, and the field $\left\{X_{j}\right\}$. In $(2.20)$ it is assumed that $B=B\left(x_{0}, r\right), x_{0} \in \Omega$, and $0<r<2 d(\Omega) ; P_{X}(E ; B)$ denotes the relative perimeter of a set $E \subset B$. We follow [18, p. 1083] to recall the definition of $P_{X}(E, B)$.

Let

$$
F(D)=\left\{\Phi=\left(\Phi_{1}, \ldots, \Phi_{n}\right) \in C_{0}^{1}\left(D, E_{n}\right):\|\Phi\|_{\infty}=\sup _{x \in D}\left(\sum_{j=1}^{n}\left|\Phi_{j}(x)\right|^{2}\right)^{1 / 2} \leq 1\right\} .
$$


We put

$$
\begin{aligned}
& \operatorname{Var}_{X}(f ; D)=\sup _{\Phi \in F(D)} \int_{D} f(x) X^{*} \Phi d x, \\
& P_{X}(E ; D)=\operatorname{Var}_{X}\left(\chi_{E} ; D\right),
\end{aligned}
$$

where $\chi_{E}$ is a characteristic function of the set $E$ and $X_{j}^{*}=\sum_{k=1}^{n} \frac{\partial}{\partial x_{k}}\left(b_{i j}(x)\right), j=$ $1, \ldots, m$.

Sobolev-type inequalities for vector fields were studied, e.g., in [11, 12, 23, 24 .

As to weighted results for vector fields, we mention the papers [21, 22], where the case of $q>p \geq 1$ was treated and a balance condition was imposed on the left and right weights in (2.15). In those papers it was assumed that the left weight belongs to the doubling class, while the right weight is of $A_{p}$-class in the metric of the field $\left\{X_{j}\right\}$.

Our result pertaining to the Sobolev inequality for vector fields $\left\{X_{j}\right\}$ reads as follows.

Theorem 2.3. Suppose $1 \leq p \leq q<\infty, \Omega \Subset E_{n}$, and $B_{0}=B\left(x_{0}, r_{0}\right)$, where $x_{0} \in \Omega$, $0<r_{0}<2 d(\Omega)$; suppose $B_{0}$ is a fixed ball in the metric (2.16) corresponding to a vector field $X=\left\{X_{j}\right\}, j=1, \ldots, m$, and satisfying condition (1.7). Assume that $v \in$ $A_{\infty}\left(B_{0}, \rho, d x\right)$ with respect to the same metric. Next, assume that the balance condition (2.4) is fulfilled in $B_{0}$ (i.e., $\Omega=B_{0}$ ).

Then in $B_{0}$ we have

$$
\begin{aligned}
& \left(\frac{1}{v\left(B_{0}\right)} \int_{B_{0}}|f(x)|^{q} v(x) d x\right)^{\frac{1}{q}} \leq \operatorname{Cr}\left(B_{0}\right)\left(\frac{1}{\left|B_{0}\right|} \int_{B_{0}} \omega(x) d x\right)^{\frac{1}{p}} \\
& \quad \times\left(\frac{1}{\left|B_{0}\right|} \int_{B_{0}} \omega^{-1 /(p-1)}(x) d x\right)^{\frac{p-1}{p}}\left(\frac{1}{\omega\left(B_{0}\right)} \int_{B_{0}}|X f|^{p} \omega(x) d x\right)^{\frac{1}{p}}
\end{aligned}
$$

for any $f \in \operatorname{Lip}_{0}\left(B_{0}\right)$. The constant $C$ depends on $n, q, C_{p q}$, the constants $C, \delta$ occurring in (2.3), and also on the compact set $\Omega$ and the field $\left\{X_{j}\right\}$.

Corollary 2.1. Under the conditions of Theorem 2.3, let $\omega \in A_{p}$, and let condition (2.5) be fulfilled instead of (2.4). Then in $B_{0}$ we have

$$
\begin{gathered}
\left(\frac{1}{v\left(B_{0}\right)} \int_{B_{0}}|f(x)|^{q} v(x) d x\right)^{1 / q} \leq \operatorname{Cr}\left(B_{0}\right)\left(\frac{1}{\omega\left(B_{0}\right)} \int_{B_{0}}|X f|^{p} \omega(x) d x\right)^{1 / p}, \\
q \geq p \geq 1, \quad f \in \operatorname{Lip}_{0}\left(B_{0}\right) .
\end{gathered}
$$

As an easy consequence, we obtain the following statement.

Corollary 2.2. Suppose $1 \leq p<Q, \omega \in A_{p}\left(B_{0}, \rho, d x\right)$, where $B_{0} \subset \Omega$ is a fixed ball in the metric (2.16). Then

$$
\begin{gathered}
\left(\frac{1}{\omega\left(B_{0}\right)} \int_{B_{0}}|f(x)|^{p Q^{\prime}} \omega(x) d x\right)^{1 / p Q^{\prime}} \leq \operatorname{Cr}\left(B_{0}\right)\left(\frac{1}{\omega\left(B_{0}\right)} \int_{B_{0}}|X f|^{p} \omega(x) d x\right)^{1 / p}, \\
1<Q<\infty, \quad f \in \operatorname{Lip}_{0}\left(B_{0}\right) .
\end{gathered}
$$

The constant $C$ only depends on $n, Q, p, C_{p}$, and on $\Omega,\left\{X_{j}\right\}$.

In our Theorem 2.3 (and in Theorem 2.4 below), the left weight belongs to a class smaller than in [22, Theorem 2], but the right weight is almost free from condition $A_{p}$. For $q=p \geq 1$, our result is new even in the case of the Euclidean metric (cf. [5. Theorem 5]). In the corresponding results, the two weights $v$ and $\omega^{-1 /(p-1)}$ were assumed to belong to the $A_{\infty}$-class [25], or to $A_{\infty}^{\beta}$ for some $\beta>n-1$ (see [5, Theorem 5]). In the 
case where $\omega \in A_{p}$, the balance condition becomes somewhat better (in [22], the balance condition looked like this:

$$
\frac{r(I)}{r(J)}\left(\frac{v(I)}{v(J)}\right)^{1 / q} \leq C\left(\frac{\omega(I)}{\omega(J)}\right)^{1 / p}
$$

for all metric balls $I, J$ with $I \subset J)$. These remarks pertain also to the following result on the Poincaré inequality.

Theorem 2.4. Suppose $1 \leq p \leq q<\infty, \Omega \Subset E_{n}$, and $B_{0}=B\left(x_{0}, r_{0}\right)$, where $x_{0} \in \Omega$, $0<r_{0} \leq 2 d(\Omega)$; suppose $B_{0}$ is a fixed ball in the metric (2.16) corresponding to a vector field $X=\left\{X_{j}\right\}$ satisfying (1.6). Assume that $v \in A_{\infty}\left(B_{0}, \rho, d x\right)$ with respect to the same metric. Next, assume that the balance condition (2.4) is fulfilled in $B_{0}$ (i.e., $\Omega:=B_{0}$ ). Then inequality (2.23) holds true with the replacement of $f$ by $f-\bar{f}_{\nu, B_{0}}$ on the left-hand side. The constant $C$ only depends on $n, q$, and $C_{p q}$, on $C$ and $\delta$ occurring in (2.3), and also on $\Omega$ and $\left\{X_{j}\right\}$.

Remark 2.3. Theorem 2.4 implies corollaries similar to Corollaries 2.1 and 2.2 , but now pertaining to the Poincaré inequality, with the replacement of $f$ by $f-\bar{f}_{\nu, B_{0}}$ on the left-hand sides of (2.24) and (2.25).

\section{§3. Proofs of Theorems 2.1 and 2.2}

Proof of Theorem 2.1. Let $f \in \operatorname{Lip}_{0}\left(B_{0}\right)$; we put $B_{0}^{+}=\left\{x \in B_{0}: f(x)>0\right\}$ and $B_{0}^{-}=$ $B_{0} \backslash \bar{B}_{0}^{+}$. Let $D^{i}$ be a connected component of $B_{0}^{+}(i=1,2, \ldots)$. We denote $D_{\alpha}=$ $\left\{x \in D^{i}: f(x)>\alpha\right\}, \alpha>0$.

Suppose that $D_{2 \alpha}$ is nonempty. Then for any fixed $x \in D_{2 \alpha}$ we can find $B(x, r(x))$ such that

$$
\left|B(x, r(x)) \backslash D_{\alpha}\right|=\gamma|B(x, r(x))|,
$$

where $\gamma \in(0,1)$ is some number independent of $\alpha, x$, and $r(x)$; this $\gamma$ will be specified later. Indeed, to prove (3.1), it suffices to put

$$
r(x)=\sup \left\{t>0:\left|B(x, t) \backslash D_{\alpha}\right| \leq \gamma|B(x, t)|\right\} .
$$

For a fixed $x \in D_{2 \alpha}$, we denote $B=B(x, r(x))$ for simplicity. Two cases are possible. 1) If

$$
\left|D_{2 \alpha} \cap B\right|<\gamma|B|,
$$

then, by (2.3), we have

$$
v\left(D_{2 \alpha} \cap B\right) \leq C \gamma^{\delta} v(B) .
$$

Next, $v(B)=v\left(B \cap D_{\alpha}\right)+v\left(B \backslash D_{\alpha}\right) \leq C \gamma^{\delta} v(B)+v\left(B \cap D_{\alpha}\right)$ by (2.3) and (3.1).

Choosing $\gamma$ so that $C \gamma^{\delta}<1$, we have

$$
v(B) \leq \frac{1}{1-C \gamma^{\delta}} v\left(B \cap D_{\alpha}\right) .
$$

Therefore, by (3.4), we obtain

$$
v\left(B \cap D_{2 \alpha}\right) \leq \frac{C \gamma^{\delta}}{1-C \gamma^{\delta}} v\left(B \cap D_{\alpha}\right)
$$

2) If

$$
\left|D_{2 \alpha} \cap B\right| \geq \gamma|B|
$$

then, by (3.1) and (3.6), we have

$$
\int_{A}\left(\int_{Z} d y\right) d x \geq \gamma^{2}|B|^{2}, \quad \text { where } A=B \backslash D_{\alpha}, \quad Z=B \cap D_{2 \alpha} \text {. }
$$


Let points $x \in A, y \in Z$ be fixed arbitrarily. Clearly, the line passing through $x$ and $y$ lies in $B$ and necessarily intersects the surfaces $\left\{x \in D^{i}: f(x)=\alpha\right\}$ and $\left\{x \in D^{i}: f(x)=2 \alpha\right\}$ at some points $x^{\prime}=x+t_{1}(y-x)$ and $x^{\prime \prime}=x+t_{2}(y-x)$, where $t_{2}>t_{1}>0$ are numbers depending on $x$ and $y$. Then $f\left(x^{\prime}\right)=\alpha$ and $f\left(x^{\prime \prime}\right)=2 \alpha$. Therefore, we have

$$
\gamma^{2}|B|^{2} \leq \int_{A}\left(\int_{Z} \frac{1}{\alpha}\left|f\left(x^{\prime}\right)-f\left(x^{\prime \prime}\right)\right| d y\right) d x
$$

whence

$$
\gamma^{2}|B|^{2} \leq \frac{1}{\alpha} \int_{A}\left(\int_{Z}\left(\int_{t_{1}(x, y)}^{t_{2}(x, y)}\left|\frac{\partial f}{\partial t}(x+t(y-x))\right| d t\right) d y\right) d x .
$$

The Fubini theorem yields

$$
\gamma^{2}|B|^{2} \leq \sum_{j=1}^{n} \frac{e_{j}(B)}{\alpha} \int_{A}\left(\int_{0}^{1}\left(\int_{\{y \in B: x+t(y-x) \in G\}}\left|\frac{\partial f}{\partial x_{j}}(x+t(y-x))\right| d y\right) d t\right) d x,
$$

where $G=B \cap\left(D_{\alpha} \backslash D_{2 \alpha}\right)$. In the inner integral we put $z=x+t(y-x)$. Then $z \in G$ and

$$
\gamma^{2}|B|^{2} \leq \sum_{j=1}^{n} \frac{e_{j}(B)}{\alpha} \int-A\left(\int_{0}^{1}\left(\int_{\{z \in G:(z-x) / t+x \in B\}}\left|\frac{\partial f}{\partial z_{j}}(z)\right| d z\right) \frac{d t}{t^{n}}\right) d x .
$$

Applying the Fubini formula once again, we obtain

$$
\begin{aligned}
\gamma^{2}|B|^{2} & \leq \sum_{j=1}^{n} \frac{e_{j}(B)}{\alpha} \int_{0}^{1}\left(\int_{G}\left|\frac{\partial f}{\partial z_{j}}\right|\left(\int_{\left\{x:\left|x_{j}-z_{j}\right|\right\} \leq t e_{j}(B)} d x\right) d z\right) \frac{d t}{t^{n}} \\
& \leq \sum_{j=1}^{n} \frac{|B| e_{j}(B)}{\alpha} \int_{G}\left|\frac{\partial f}{\partial z_{j}}\right| d z
\end{aligned}
$$

whence

$$
1 \leq \sum_{j=1}^{n}\left(\frac{n^{\frac{q-1}{q}} e_{j}(B)}{|B| \alpha \gamma^{2}} \int_{G}\left|\frac{\partial f}{\partial z_{j}}\right| d z\right)^{q} .
$$

By the Hölder inequality, this implies

$$
1 \leq \sum_{j=1}^{n}\left(\frac{n^{\frac{q-1}{q}} e_{j}(B)}{|B| \alpha \gamma^{2}}\right)^{q}\left(\int_{B} \omega_{j}^{-1 /(p-1)}(x) d x\right)^{q(p-q) / p}\left(\int_{G}\left|\frac{\partial f}{\partial z_{j}}\right|^{p} \omega_{j}(z) d z\right)^{q / p}
$$

Estimates (2.7) and (3.8) show that

$$
1 \leq \frac{n^{q-1} A_{p q}^{q}}{\gamma^{2 q} \alpha^{q}} \frac{1}{v(B)} \sum_{j=1}^{n}\left(\int_{G}\left|\frac{\partial f}{\partial z_{j}}\right|^{p} \omega_{j}(z) d z\right)^{q / p} .
$$

Consequently,

$$
v\left(B \cap D_{2 \alpha}\right) \leq \frac{n^{q-1} A_{p q}^{q}}{\gamma^{2 q} \alpha^{q}} \sum_{j=1}^{n}\left(\int_{G}\left|\frac{\partial f}{\partial z_{j}}\right|^{p} \omega_{j}(z) d z\right)^{q / p} .
$$

By (3.5) and (3.10), we have

$$
\begin{aligned}
v\left(B \cap D_{2 \alpha}\right) \leq & \frac{C \gamma^{\delta}}{1-C \gamma^{\delta}} v\left(B \cap D_{\alpha}\right) \\
& +\frac{n^{q-1} A_{p q}^{q}}{\gamma^{2 q} \alpha^{q}} \sum_{j=1}^{n}\left(\int_{B \cap\left(D_{\alpha} \backslash D_{2 \alpha}\right)}\left|\frac{\partial f}{\partial z_{j}}\right|^{p} \omega_{j}(z) d z\right)^{q / p} .
\end{aligned}
$$


The system of balls $\left\{B=B(x, r(x)): x \in D_{2 \alpha}\right\}$ covers $D_{2 \alpha}$. By the GuzmánBesicovitch lemma on comparable intervals (parallelepipeds), see [14, the system $\{B\}$ admits a finite or countable subfamily $\left\{B_{i}\right\}_{i=1}^{\infty}$ that covers $D_{2 \alpha}$ and has finite multiplicity, i.e.,

$$
\sum_{i=1}^{\infty} \chi_{B_{i}}(x) \leq C_{n}
$$

where $C_{n}>0$ depends only on $n$. Summing over $j$ all inequalities

$$
\begin{gathered}
v\left(B_{j} \cap D_{2 \alpha}\right) \leq \frac{C \gamma^{\delta}}{1-C \gamma^{\delta}} v\left(B_{j} \cap D_{\alpha}\right)+\frac{n^{q-1} A_{p q}^{q}}{\gamma^{2 q} \alpha^{q}} \sum_{k=1}^{n}\left(\int_{G_{j}}\left|\frac{\partial f}{\partial z_{k}}\right|^{p} \omega_{k}(z) d z\right)^{q / p}, \\
G_{j}=B_{j} \cap\left(D_{\alpha} \backslash D_{2 \alpha}\right),
\end{gathered}
$$

obtained from (3.11) for $B=B_{j}$, and using (3.12), we arrive at

$$
v\left(D_{2 \alpha}\right) \leq \frac{C_{n} C \gamma^{\delta}}{1-C \gamma^{\delta}} v\left(D_{\alpha}\right)+\frac{C_{n} n^{q-1} A_{p q}^{q}}{\gamma^{2 q} \alpha^{q}} \sum_{j=1}^{n}\left(\int_{D_{\alpha} \backslash D_{2 \alpha}}\left|\frac{\partial f}{\partial z_{j}}\right|^{p} \omega_{j}(z) d z\right)^{q / p} .
$$

We integrate $(3.13)$ over $(0, \infty)$ :

$$
\begin{aligned}
\int_{0}^{\infty} v\left(D_{2 \alpha}\right) d \alpha^{q} \leq & \frac{C_{n} C \gamma^{\delta}}{1-C \gamma^{\delta}} \int_{0}^{\infty} v\left(D_{\alpha}\right) d \alpha^{q} \\
& +\sum_{j=1}^{n} \frac{C_{n} n^{q-1} A_{p q}^{q}}{\gamma^{2 q}} \int_{0}^{\infty} \frac{d \alpha}{\alpha}\left(\int_{D_{\alpha} \backslash D_{2 \alpha}}\left|\frac{\partial f}{\partial z_{j}}\right|^{p} \omega_{j}(z) d z\right)^{q / p}
\end{aligned}
$$

Since

$$
\int_{0}^{\infty} v\left(D_{2 \alpha}\right) d \alpha^{q}=\frac{1}{2^{q}} \int_{D^{i}} f^{q}(x) v(x) d x, \quad \int_{0}^{\infty} v\left(D_{\alpha}\right) d \alpha^{q}=\int_{D^{i}} f^{q}(x) v(x) d x,
$$

we can use the Minkowski inequality to show that

$$
\begin{aligned}
\left(\frac{1}{2^{q}}\right. & \left.-\frac{C_{n} C \gamma^{\delta}}{1-C \gamma^{\delta}}\right) \int_{D^{i}} f^{q}(x) v(x) d x \\
& \leq \frac{C_{n} q n^{q-1} A_{p q}^{q}}{\gamma^{2 q}} \sum_{j=1}^{n}\left(\int_{D^{i}}\left|\frac{\partial f}{\partial z_{j}}\right|^{p} \omega_{j}(z)\left(\int_{f(z) / 2}^{f(z)} \frac{d \alpha}{\alpha}\right)^{p / q} d z\right)^{q / p} .
\end{aligned}
$$

Choosing $\gamma$ so small that

$$
\frac{1}{2^{q}}-\frac{C_{n} C \gamma^{\delta}}{1-C \gamma^{\delta}}>0
$$

we see that (3.15) implies that

$$
\begin{aligned}
\int_{D^{i}} f^{q}(x) v(x) d x \leq & \left(\frac{1}{2^{q}}-\frac{C_{n} C \gamma^{\delta}}{1-C \gamma^{\delta}}\right)^{-1} \frac{C_{n} q n^{q-1} \ln 2}{\gamma^{2 q}} A_{p q}^{q} \\
& \times \sum_{j=1}^{n}\left(\int_{D^{i}}\left|\frac{\partial f}{\partial z_{j}}\right|^{p} \omega_{j}(z) d z\right)^{q / p} .
\end{aligned}
$$

Summing the inequalities (3.17) for all $D^{i}$, we obtain

$$
\begin{aligned}
\int_{B_{0}^{+}} f^{q}(x) v(x) d x & \leq C_{0}^{q} A_{p q}^{q} \sum_{j=1}^{n}\left(\int_{B_{0}^{+}}\left|\frac{\partial f}{\partial z_{j}}\right|^{p} \omega_{j}(z) d z\right)^{q / p}, \\
C_{0}^{q} & =\left(\frac{1}{2^{q}}-\frac{C_{n} C \gamma^{\delta}}{1-C \gamma^{\delta}}\right)^{-1} \frac{C_{n} q n^{q-1}}{\gamma^{2 q}} \ln 2 .
\end{aligned}
$$


A similar inequality holds true in $B_{0}^{-}$for the function $-f(x)$ :

$$
\int_{B_{0}^{-}}(-f(x))^{q} v(x) d x \leq C_{0}^{q} A_{p q}^{q} \sum_{j=1}^{n}\left(\int_{B_{0}^{-}}\left|\frac{\partial f}{\partial z_{j}}\right|^{p} \omega_{j}(z) d z\right)^{q / p} .
$$

Estimates (3.18) and (3.19) imply (2.8).

Theorem 2.1 is proved.

Proof of Theorem 2.2. We can find $A \in E_{1}$ such that

$$
\left|x \in B_{0}: f(x)>A\right| \leq \frac{1}{2}\left|B_{0}\right| \leq\left|x \in B_{0}: f(x) \geq A\right| .
$$

Put $B_{0}^{+}=\left\{x \in B_{0}: f(x)>A\right\}$. Let $D^{i}(i=1,2, \ldots)$ be a connected component of $B_{0}^{+}$. We denote $D_{\alpha}=\left\{x \in D^{i}: f(x)>A+\alpha\right\}, \alpha>0$. Then $\left|B_{0} \backslash B_{0}^{+}\right| \geq \frac{1}{2}\left|B_{0}\right|$ and $\left|B_{0} \backslash B_{0}^{-}\right| \geq \frac{1}{2}\left|B_{0}\right|$. Let $\alpha>0$ be such that $D_{2 \alpha}$ is nonempty. For any fixed $x \in D_{2 \alpha}$, there is a ball $B(x, r(x))$ such that

$$
\left|\left(B(x, r(x)) \cap B_{0}\right) \backslash D_{\alpha}\right|=\gamma\left|B(x, r(x)) \cap B_{0}\right|,
$$

where $0<\gamma<\frac{1}{2}$ is a sufficiently small number to be specified later. Indeed, it suffices to take

$$
r(x)=\sup \left\{t>0:\left|\left(B(x, t) \cap B_{0}\right) \backslash D_{\alpha}\right| \leq \gamma\left|B(x, t) \cap B_{0}\right|\right\} .
$$

Let a ball $B=B(x, r(x))$ in the system $\left\{B=B(x, r(x)): x \in D_{2 \alpha}\right\}$ be fixed. If a)

$$
\left|D_{2 \alpha} \cap B\right|<\gamma\left|B \cap B_{0}\right|,
$$

then

$$
v\left(D_{2 \alpha} \cap B\right) \leq C \gamma^{\delta} v\left(B \cap B_{0}\right) .
$$

On the other hand, (3.20) and (2.6) imply

$$
v\left(B \cap B_{0}\right)=v\left(\left(B \cap B_{0}\right) \backslash D_{\alpha}\right)+v\left(B \cap D_{\alpha}\right) \leq C \gamma^{\delta} v\left(B \cap B_{0}\right)+v\left(B \cap D_{\alpha}\right) .
$$

Consequently, if $\gamma$ satisfies $C \gamma^{\delta}<1$, then

$$
v\left(B \cap B_{0}\right) \leq \frac{1}{1-C \gamma^{\delta}} v\left(B \cap D_{\alpha}\right),
$$

and $\left(3.21^{\prime}\right)$ shows that

$$
v\left(B \cap D_{2 \alpha}\right) \leq \frac{C \gamma^{\delta}}{1-C \gamma^{\delta}} v\left(B \cap D_{\alpha}\right)
$$

Now, if

$$
\left|D_{2 \alpha} \cap B\right| \geq \gamma\left|B \cap B_{0}\right|,
$$

then we can repeat all the arguments in Theorem 2.1.

In this case, (3.20) and (3.23) imply that

$$
\left|D_{2 \alpha} \cap B\right|>\varepsilon \gamma|B|, \quad\left|\left(B \cap B_{0}\right) \backslash D_{\alpha}\right|>\varepsilon \gamma|B|,
$$

where $\varepsilon \in(0,1)$ is a number depending on $n$. Next, arguing as in Theorem 2.1 and using (3.24) and (2.9), we obtain

$$
\left(\int_{B_{0}}|f(x)-A|^{q} v(x) d x\right)^{1 / q} \leq C_{0} A_{p q} \sum_{j=1}^{n}\left(\int_{B_{0}}\left|\frac{\partial f}{\partial x_{j}}\right|^{p} \omega_{j}(x) d x\right)^{1 / p} .
$$

We show that

$$
\left\|f-\bar{f}_{v, B_{0}}\right\|_{q, v} \leq 2\|f-A\|_{q, v} .
$$


Indeed, to obtain (3.26) it suffices to apply the Minkowski inequality,

$$
\begin{aligned}
\left\|f-\bar{f}_{v, B_{0}}\right\|_{q, v} & \leq\|f-A\|_{q, v}+\left\|\bar{f}_{v, B_{0}}-A\right\|_{q, v} \\
& =\|f-A\|_{q, v}+\left|\bar{f}_{v, B_{0}}-A\right|\left(v\left(B_{0}\right)\right)^{1 / q},
\end{aligned}
$$

and the Hölder inequality,

$$
\left|\bar{f}_{v, B_{0}}-A\right| \leq \frac{1}{v\left(B_{0}\right)} \int_{B_{0}}|f-A| v(x) d x \leq v\left(B_{0}\right)^{-1 / q}\|f-A\|_{q, v} .
$$

Theorem 2.2 is proved.

Proof of the statement of Example 2.1. We apply Theorem 2.1 in the case where $p=2$, $v(x) \equiv 1, \omega_{j}(x)=|x|_{\bar{\sigma}}^{\beta_{j}}, \sigma_{j}=\frac{\beta_{j}+\eta}{2}, j=1, \ldots, n$. It suffices to check condition (2.7), because (2.3) is fulfilled obviously. We consider two cases separately: 1) $\rho(a) \leq C R$, and 2) $\rho(a)>C R$, where $C>1$ is a sufficiently large number independent of $R$ and $a$. If 1$)$ is fulfilled, then, for any ball $B=B(x, r(x))$, where $x \in B(a, R), r<R$, there are two possibilities: a) $\rho(x)<C r$, or b) $\rho(a)>C r(C$ is as in 1$)$ ). In the case of 1a), we can verify $(2.7)$ for $p=2, j=1, \ldots, n$ :

$$
(v(B))^{1 / q}|B|^{-1} e_{j}\left(B\left(\int_{B} \omega_{j}^{-1}(x) d x\right)^{1 / 2} \leq|B(x, r)|^{1 / q-1} r^{\sigma_{j}}\left(\int_{B(x, r)}|y|_{\bar{\sigma}}^{-\beta_{j}} d y\right)^{1 / 2} .\right.
$$

Observe that $\frac{1}{n}|y|_{\bar{\sigma}} \leq \rho(y) \leq|y|_{\bar{\sigma}} ;$ consequently,

$$
\int_{B(x, r)}|y|_{\bar{\sigma}}^{-\beta_{j}} d y \leq \int_{B(x, r)} \rho(y)^{-\beta_{j}} d y \leq C r^{\sum_{k=1}^{n} \sigma_{k}-\beta_{j}} .
$$

Since $\sigma_{j}=\frac{\beta_{j}+\eta}{2}$, the right-hand side of (3.27) is dominated by

$$
|B(x, r)|^{1 / q-1 / 2+\left(2 \sigma_{j}-\beta_{j}\right) /\left(2 \sum_{k=1}^{n} \sigma_{k}\right)}=C|B(x, r)|^{1 / q-1 / 2+\eta /\left(2 \sum_{k=1}^{n} \sigma_{k}\right)} \leq C_{2} .
$$

In the case of $1 \mathrm{~b})$, the left-hand side of (3.27) does not exceed

$$
r^{\sigma_{j}}|B(x, r)|^{1 / q-1 / 2}|x|_{\bar{\sigma}}^{-\beta_{j} / 2} \leq C_{2}
$$

because

$$
|x|_{\bar{\sigma}}^{-\beta_{j} / 2} \leq \rho(x)^{-\beta_{j} / 2} \leq C_{3} r^{-\beta_{j} / 2} .
$$

Case 2) is similar to case b): for any ball $B(x, r)$, where $x \in B(a, R), r<R$, the left-hand side of (3.27) is estimated by the expression

$$
C|B(x, r)|^{1 / q-1 / 2} R^{\sigma_{j}-\beta_{j} / 2} \leq C_{3},
$$

where $C, C_{2}$, and $C_{3}$ depend on $n, \beta$, and $\eta$. Using (2.11), we see that this implies $(2.12)$.

Proof of the statement of Example 2.2. We apply Theorem 2.2 to the case of $v(x) \equiv 1$, $\omega_{j}(x)=|x|_{\bar{\sigma}}^{\beta_{j}}, \sigma_{j}=\frac{\beta_{j}+\eta}{2}, p=2$. It suffices to check (2.6) and (2.9). Condition (2.6) is fulfilled obviously, and condition (2.9) was verified in Example 2.1. 


\section{§4. Properties of Metric Balls of a Vector Field}

Let $X=\left\{X_{j}\right\}, j=1, \ldots, n$, be a fixed vector field of the same form and satisfying the same assumptions as in $\S 2$. Let $\rho(x, y)$ be the metric corresponding to this field. We assume that condition (1.7) is fulfilled and that the coefficients of the field are infinitely differentiable.

We need several auxiliary statements.

Lemma 4.1. Suppose $\Omega \Subset E_{n}$ is a compact set, and $B_{0}=B\left(x_{0}, r_{0}\right)$, where $x_{0} \in \Omega, r_{0} \leq$ $5 d(\Omega)$. Let $B=B(x, r)$ be another ball, $x \in B_{0}, r<2 r_{0}$. Then there exists $z \in B_{0} \cap B$ such that $B(z, r / 4) \subset B_{0} \cap B$.

Proof of Lemma 4.1. There exists a continuous curve $\left\{\gamma_{x_{o} x}\right\}$ that connects $x_{0}$ and $x$ and satisfies (2.19). If i) $r / 2 \leq \rho\left(x_{0}, x\right)$, then we choose $z \in\left\{\gamma_{x_{o} x}\right\}$ so that $\rho(z, x)=r / 2$, i.e., $z \in \partial B(x, r / 2)$. Then we have $\rho\left(z, x_{0}\right)=r_{0}-r / 2$ by $(2.19)$. We show that $B(z, r / 2) \subset$ $B \cap B_{0}$. Indeed, by the triangle inequality, for any $y \in \partial B_{0}$ we have

$$
\rho(z, y) \geq \rho\left(y, x_{0}\right)-\rho\left(x_{0}, z\right)=r_{0}-\left(r_{0}-\frac{r}{2}\right)=\frac{r}{2} .
$$

Therefore, $\rho(z, y) \geq \frac{r}{2}$ whenever $y \in \partial B$, and we have

$$
\rho(z, t) \geq \rho(t, x)-\rho(x, z)=r-\frac{r}{2}=\frac{r}{2},
$$

i.e., $\rho(z, t) \geq \frac{r}{2}$ for all $t \in \partial B$.

Now we show that $\rho\left(z, \partial\left(B_{0} \cap B\right)\right) \geq r / 2$. This means that

$$
B(z, r / 2) \subset B_{0} \cap B \text {. }
$$

If ii) $r / 2 \geq \rho\left(x_{0}, x\right)$, then we choose $z \in\left\{\gamma_{x_{o} x}\right\}$ such that $\rho(z, x)=\rho\left(x_{0}, x\right) / 2$ (i.e., $\left.z \in \partial B\left(x, \rho\left(x_{0}, x\right) / 2\right)\right)$. Then $\rho\left(x_{0}, z\right)=\rho(x, z)=\rho\left(x_{0}, x\right) / 2$ by (2.19). Now we check that $B(z, r / 4) \subset B_{0} \cap B$. For $y \in \partial B_{0}$, by the triangle inequality we have $\rho(z, y) \geq$ $\rho\left(x_{0}, y\right)-\rho\left(x_{0}, x\right) / 2 \geq r_{0}-r / 4 \geq r / 4$, and also for $t \in \partial B$ we have $\rho(z, t) \geq \rho(x, t)-$ $\rho(x, z) \geq r-\rho\left(x_{0}, z\right) / 2 \geq r-r / 4 \geq 3 r / 4$. Therefore, $\rho\left(z, \partial\left(B_{0} \cap B\right)\right) \geq r / 4$, which means that

$$
B(z, r / 4) \subset B_{0} \cap B .
$$

Relations (4.1) and (4.2) imply the claim.

Lemma 4.1 is proved.

Lemma 4.2 (of Besicovitch type). Suppose $\Omega \Subset E_{n}$, and $A$ is a bounded subset in $\Omega$. If a ball $B(x, r(x))$ is given for any $x \in A$, i.e., $A \subset \bigcup_{x \in A} B(x, r(x))$, then there exists a finite or countable family of balls $\left\{B\left(x_{j}, r_{j}\right)\right\}_{j=1}^{\infty}$, where $r_{j}=r\left(x_{j}\right)$, such that

$$
A \subset \bigcup_{j=1}^{\infty} B\left(x_{j}, r_{j}\right), \quad \sum_{j=1}^{\infty} \chi_{B\left(x_{j}, r_{j}\right)}(x) \leq C,
$$

where $C$ depends on $n$, the field $\left\{X_{j}\right\}$, and $\Omega$.

Proof. As in [14], we denote

$$
r_{1}^{*}=\sup \{r(B): B=B(x, r(x)), x \in A\} .
$$

Then there exists $B\left(x_{1}, r_{1}\right)$ with $r_{1}>r_{1}^{*} /(1+\delta)$, where $\delta>0$ is an arbitrary fixed number. We put $B_{1}=B\left(x_{1}, r_{1}\right)$. Suppose that $m$ balls $B_{1}, \ldots, B_{m}$ have already been chosen. We put

$$
r_{m+1}^{*}=\sup \left\{r(B): B=B(x, r(x)), x \in A \backslash\left(\bigcup_{k=1}^{m} B_{k}\right)\right\} .
$$


Then there exists $B\left(x_{m+1}, r_{m+1}\right)$ with $r_{m+1} \geq r_{m+1}^{*} /(1+\delta)$, and we set $B_{m+1}=$ $B\left(x_{m+1}, r_{m+1}\right)$.

We argue as in [14] to show that the system $\left\{B_{j}\right\}_{j=1}^{\infty}$ satisfies (4.3). Observe that the balls $B\left(x_{j}, \frac{1}{2} r_{j}\right)$ are pairwise disjoint. Therefore, by (2.18), we have

$$
|A| \geq \sum_{j=1}^{\infty}\left|\left(B\left(x_{j}, \frac{1}{3} r_{j}\right)\right)\right| \geq C \sum_{j=1}^{\infty} r_{j}^{Q},
$$

whence we see that $r\left(B_{j}\right) \rightarrow 0$ as $j \rightarrow \infty$. Suppose $\bar{x} \in A$, but $\bar{x} \notin \bigcup_{j=1}^{\infty} B_{j}$. Then there exists $B\left(x_{i}, r_{i}\right)$ with $r(\bar{x})>r_{i}$, where $B(\bar{x}, r(\bar{x}))$ is the ball in the initial covering. This means that the point $\bar{x}$ was missed in the process of choosing the points $\bar{x}$, which is impossible. Thus, $A \subset \bigcup_{j=1}^{\infty} B_{j}$.

Now we show that, at each point $z \in E_{n}$, only finitely many balls of the system can intersect at $z$, and moreover, their number is controlled in terms of the field, $\Omega$, and $n$.

Let $\gamma_{x y}(t)$ be a geodesic that connects $x, y \in E_{n}$, i.e., $\gamma(t)$ satisfies (2.19). For $\varepsilon>0$ and $x_{0}, z \in E_{n}$, we denote

$$
K\left(z, \varepsilon,\left\{\gamma_{x_{o} z}\right\}\right)=\bigcup_{0<t<\rho\left(z, x_{0}\right)}\left[B\left(\gamma_{z x_{0}}(t), \varepsilon t\right) \cap S(z, t)\right] ;
$$

this set will be called the metric cone of opening $\varepsilon$, with vertex at $z$, and with ruling $\gamma_{z x_{0}}(t)$.

Let $z, x_{0} \in \Omega$. We shall show that $z$ can serve as the vertex for only finitely many cones (4.4) such that they cover the ball $B\left(z, \rho\left(z, x_{0}\right)\right)$ and the cones of smaller opening $\frac{\varepsilon}{C}(C$ does not depend on $\varepsilon$ ) do not intersect (i.e., have no common points except for $z$ ). If this is not true, then

$$
|B(x, t)| \geq \sum_{s=1}^{N}\left|K\left(z, \varepsilon / C,\left\{\gamma_{z x_{s}}\right\}\right)\right|, \quad x_{s} \in B(z, t),
$$

where $N$ is the minimal number of such cones. The cone $K\left(z, \varepsilon / C,\left\{\gamma_{z x_{s}}\right\}\right)$ contains a ball $B(a, t \varepsilon / 2)$, where $a \in\left\{\gamma_{z x_{s}}\right\}$. By (2.17), we have $|B(a, \varepsilon t / 2)| \geq(1 / C(\varepsilon))|B(z, t)|$, so that $|B(z, t)| \geq(1 / C(\varepsilon)) N|B(z, t)|$. It follows that $N / C(\varepsilon)<1$, i.e., the number of the cones is bounded by the number $C(\varepsilon)$.

Any fixed cone $K=K\left(z, \varepsilon,\left\{\gamma_{z x_{0}}\right\}\right)$ possesses the following property: if $x_{1}, x_{2} \in K$ and

$$
\rho\left(x_{1}, z\right) \leq \rho\left(x_{2}, z\right) \leq(1+\delta) \rho\left(x_{1}, z\right)
$$

then

$$
\rho\left(x_{1}, x_{2}\right) \leq \rho\left(x_{1}, z\right) .
$$

Indeed, we can find $t_{1}, t_{2} \in\left\{\gamma_{z x_{0}}\right\}$ with $\rho\left(x_{1}, z\right)=t_{1}$ and $\rho\left(x_{2}, z\right)=t_{2}$. Therefore,

$$
\rho\left(x_{1}, x_{2}\right) \leq \rho\left(x_{1}, t_{1}\right)+t_{2}-t_{1}+\rho\left(x_{2}, t_{2}\right) \leq \varepsilon t_{1}+t_{2}-t_{1}+\varepsilon t_{2} \leq 2(\varepsilon+\delta) t_{1} .
$$

Choosing $\delta=\varepsilon$ and $4 \varepsilon<1$, we have

$$
\rho\left(x_{1}, x_{2}\right) \leq \min \left(\rho\left(x_{1}, z\right), \rho\left(x_{2}, z\right)\right) .
$$

Hence, any ball centered at $x_{1}\left(x_{2}\right)$ and containing $z$ must also contain the point $x_{2}\left(x_{1}\right)$. This means that the point $z$ lies in at most one of the balls in $\left\{B_{j}\right\}_{j=1}^{\infty}$ centered at a point of $K$. By construction, the center of any consequent ball belongs to none of the preceding balls. But, if $x_{1}$ is the center of the $i$ th ball and $x_{2}$ is the center of the $j$ th ball, then (4.5) implies that $x_{1} \in B_{j}$ and $x_{2} \in B_{i}$, a contradiction.

The lemma is proved. 


\section{§5. Proofs of Theorems 2.3 And 2.4}

Proof of Theorem 2.3 (of Sobolev type). We argue as in Theorem 2.1, keeping the entire notation of that theorem concerning $\alpha, D_{\alpha}, B_{0}$, and $B$. Let $0<\gamma<1 / C_{1}^{3}$ be fixed; here $C_{1}$ is the constant occurring in (2.17). By applying (2.17), it is easy to show that for any $x \in D_{2 \alpha}$ there exists $B=B(x, r(x))$ such that $r(x) \in\left(0,4 r\left(B_{0}\right)\right)$ and (3.1) is fulfilled. Indeed, the metric topology in question coincides with the Euclidean topology in $E_{n}$. Therefore, $B(x, t) \backslash D_{\alpha}=\varnothing$ for all sufficiently small $t$. Also, we have a continuous map $\left(E_{n}, \rho\right) \leftrightarrow\left(E_{n},|\cdot|\right)$ between the metric space and the Euclidean space [18. Let $x \in D_{2 \alpha}$, let $y$ be a point on the surface of the ball $B\left(x, 4 r\left(B_{0}\right)\right)$, and let $\left\{\gamma_{x y}\right\}$ be a geodesic connecting $x$ and $y$ (i.e., this curve satisfies (2.19)). Let $z$ be a point of $\partial B\left(y, r\left(B_{0}\right)\right) \cap\left\{\gamma_{x y}\right\}$. Then $B\left(z, r\left(B_{0}\right)\right) \subset B\left(x, 4 r\left(B_{0}\right)\right) \backslash B\left(x, 2 r\left(B_{0}\right)\right)$ because $\rho(z, x)=$ $3 r\left(B_{0}\right)$. Consequently, putting $t=4 r\left(B_{0}\right)$ and using (2.17), we obtain $\left|B(x, t) \backslash D_{\alpha}\right| \geq$ $\left|B\left(z, r\left(B_{0}\right)\right)\right| \geq \frac{1}{C_{1}^{3}}\left|B\left(z, 8 r\left(B_{0}\right)\right)\right| \geq \frac{1}{C_{1}^{3}}|B(x, t)|$, because $B\left(z, 4 r\left(B_{0}\right)\right) \subset B\left(z, 8 r\left(B_{0}\right)\right)$. Then $\left|B\left(x, 4 r\left(B_{0}\right)\right) \backslash D_{\alpha}\right| \geq \gamma\left|B\left(x, 4 r\left(B_{0}\right)\right)\right|$. Thus, there exists $r(x) \in\left(0,4 r\left(B_{0}\right)\right)$ such that (3.1) is fulfilled. Let $B=B(x, r(x))$. If 1$)\left|B \cap D_{2 \alpha}\right|<\gamma|B|$, then, as in Theorem 2.1, we have

$$
v\left(B \cap D_{2 \alpha}\right) \leq \frac{C \gamma^{\delta}}{1-C \gamma^{\delta}} v\left(B \cap D_{\alpha}\right) .
$$

If 2) $\left|D_{2 \alpha} \cap B\right| \geq \gamma|B|$, then, by Federer's formula [26], we have

$$
\int_{B \cap\left(D_{\alpha} \backslash D_{2 \alpha}\right)}|X f| d x=\int_{\alpha}^{2 \alpha} P_{X}\left(E_{t} ; B\right) d t
$$

where $E_{t}=\{x \in B: f(x)>t\}, t \in(\alpha, 2 \alpha)$, and $P_{X}\left(E_{t} ; B\right)$ is the perimeter of $E_{t}$ relative to $B$ (see (2.22)). Next we use the following local isoperimetric inequality for the ball $B$ :

$$
P_{X}\left(E_{t} ; B\right) \geq C \frac{|B|}{r(B)}, \quad t \in(\alpha, 2 \alpha),
$$

where $C$ is independent of $B, r(B), t$, and $E_{t}$. Estimate (5.3) follows from inequality (2.20) (which was proved, e.g., in [18, 22]). Putting $E=E_{t}$ and using the estimates

$$
\left|E_{t} \cap B\right| \geq \gamma|B|, \quad\left|B \backslash E_{t}\right| \geq \gamma|B|, \quad t \in(\alpha, 2 \alpha),
$$

from (5.2) and (5.3) we deduce the inequality

$$
\int_{B \cap\left(D_{\alpha} \backslash D_{2 \alpha}\right)}|X f| d x \geq C \frac{|B|}{r(B)} \alpha .
$$

This implies that

$$
1 \leq\left(\frac{r(B)}{|B| \alpha} \int_{\left(D_{\alpha} \backslash D_{2 \alpha}\right) \cap B}|X f| d x\right)^{q} .
$$

This inequality replaces estimate (3.7) in the proof of Theorem 2.1, and the rest of that proof needs no modification. By the Hölder inequality,

$$
1 \leq\left(\frac{r(B)}{C|B| \alpha}\right)^{q}\left(\int_{B} \omega^{-1 /(p-1)}(x) d x\right)^{(p-1) q / p}\left(\int_{\left(D_{\alpha} \backslash D_{2 \alpha}\right) \cap B}|X f|^{p} \omega(x) d x\right)^{q / p}
$$

and, by the balance condition (2.4),

$$
v\left(B \cap D_{2 \alpha}\right) \leq\left(\frac{1}{C \alpha}\right)^{q}\left(\frac{r\left(B_{1}\right)}{\left|B_{1}\right|}\right)^{q} v\left(B_{1}\right)\left(\omega^{-1 /(p-1)}\left(B_{1}\right)\right)^{q(p-1) / p} .
$$


Now, using (2.17) and the properties of $v$ and $\omega$, we arrive at estimate (5.7) with $B_{1}$ replaced by $B_{0}$. Therefore, recalling (3.1), we obtain

$$
\begin{aligned}
v\left(B \cap D_{2 \alpha}\right) \leq & \frac{C \gamma^{\delta}}{1-C \gamma^{\delta}} v\left(B \cap D_{\alpha}\right)+\frac{C C_{p q}^{q}}{\alpha^{q}}\left(\omega^{-1 /(p-1)}\left(B_{0}\right)\right)^{q(p-1) / p} \\
& \times\left(\frac{r\left(B_{0}\right)}{\left|B_{0}\right|}\right)^{q} v\left(B_{0}\right)\left(\int_{B \cap\left(D_{\alpha} \backslash D_{2 \alpha}\right)}|X f|^{p} \omega(x) d x\right)^{q / p} .
\end{aligned}
$$

The system of metric balls $\left\{B=B(x, r(x)): x \in D_{2 \alpha}\right\}$ covers $D_{2 \alpha}$. By Lemma 4.2, there exists a subfamily $\left\{B_{i}\right\}_{i=1}^{\infty}$ such that

$$
\sum_{i=1}^{\infty} \chi_{B_{i}}(x) \leq C, \quad D_{2 \alpha} \subset \bigcup_{i=1}^{\infty} B_{i},
$$

where $C>0$ depends on $n, \Omega$, and the field $\left\{X_{j}\right\}$. Arguing as in Theorem 2.1, we get

$$
\begin{aligned}
v\left(D_{2 \alpha}\right) \leq \frac{C_{n} C \gamma^{\delta}}{1-C \gamma^{\delta}} v\left(D_{\alpha}\right) & +C_{n} C C_{p q}^{q}\left(\omega^{-1 /(p-1)}\left(B_{0}\right)\right)^{q(p-1) / p} \\
& \times\left(\frac{r\left(B_{0}\right)}{\left|B_{0}\right|}\right)^{q} v\left(B_{0}\right)\left(\int_{D_{\alpha} \backslash D_{2 \alpha}}|X f|^{p} \omega(x) d x\right)^{q / p} .
\end{aligned}
$$

Now we integrate (5.10) and choose $\gamma$ sufficiently small, obtaining

$$
\begin{aligned}
\int_{B_{0}^{+}} f^{q}(x) v(x) d x \leq & C C_{p q}^{q}\left(\frac{r\left(B_{0}\right)}{\left|B_{0}\right|}\right)^{q} v\left(B_{0}\right)\left(\omega^{-1 /(p-1)}\left(B_{0}\right)\right)^{\frac{q(p-1)}{p}} \\
& \times\left(\int_{B_{0}^{+}}|X f|^{p} \omega(x) d x\right)^{q / p} .
\end{aligned}
$$

A similar inequality is valid in $B_{0}^{-}$for the function $-f(x)$. As a result, we have

$\int_{B_{0}}|f(x)|^{q} v(x) d x \leq C C_{p q}^{q}\left(\frac{r\left(B_{0}\right)}{\left|B_{0}\right|}\right)^{q} v\left(B_{0}\right)\left(\omega^{-1 /(p-1)}\left(B_{0}\right)\right)^{\frac{q(p-1)}{p}}\left(\int_{B_{0}}|X f|^{p} \omega(x) d x\right)^{q / p}$.

The theorem is proved.

Proof of Theorem 2.4. We modify somewhat the argument at the beginning of the proof of Theorem 2.2.

Let $r(x)$ and $B(x, r(x))$ be chosen as in (3.20). If a) $\left|D_{2 \alpha} \cap B\right|<\gamma\left|B \cap B_{0}\right|$, then, since $v \in A_{\infty}$, we obtain $v\left(D_{2 \alpha} \cap B\right) \leq C \gamma^{\delta} v(B)$. Next, we have $v(B) \leq C_{2} v\left(B \cap B_{0}\right)$, whence $v\left(B \cap D_{2 \alpha}\right) \leq C C_{2} \gamma^{\delta} v\left(B \cap B_{0}\right)$, where $C_{2}>0$ depends on the constants $C, \delta$ in (2.3) and on $n, \Omega,\left\{X_{j}\right\}$.

Similarly, (3.20) implies $v\left(\left(B \cap B_{0}\right) \backslash D_{2 \alpha}\right) \leq C C_{2} \gamma^{\delta} v\left(B \cap B_{0}\right)$. Then $v\left(B \cap B_{0}\right)=$ $v\left(\left(B \cap B_{0}\right) \backslash D_{\alpha}\right)+v\left(B \cap D_{\alpha}\right) \leq C C_{2} \gamma^{\delta} v\left(B \cap B_{0}\right)+v\left(B \cap D_{\alpha}\right)$. Therefore, if $\gamma$ satisfies $C C_{2} \gamma^{\delta}<1$, then

whence

$$
v\left(B \cap B_{0}\right) \leq \frac{1}{1-C C_{2} \gamma^{\delta}} v\left(B \cap D_{\alpha}\right),
$$

$$
v\left(B \cap D_{2 \alpha}\right) \leq \frac{C \gamma^{\delta}}{1-C \gamma^{\delta}} v\left(B \cap D_{\alpha}\right) .
$$

In the case where b) $\left|D_{2 \alpha} \cap B\right| \geq \gamma\left|B \cap B_{0}\right|$, we argue as in Theorem 2.1. By the isoperimetric estimate $(2.20)$ with $E=E_{t}$, we have

$$
P_{X}\left(E_{t} ; B\right) \geq C \frac{|B|^{1 / Q}}{r(B)}\left|B \cap B_{0}\right|^{\frac{Q-1}{q}},
$$


where $t \in(\alpha, 2 \alpha)$. Thus, an estimate similar to (5.4) takes the form

$$
\int_{B \cap\left(D_{\alpha} \backslash D_{2 \alpha}\right)}|X f| d x \geq C \alpha \frac{|B|^{1 / Q}}{r(B)}\left|B_{0} \cap B\right|^{\frac{Q-1}{q}} .
$$

Now, applying Lemma 4.1, we obtain $\left|B_{0} \cap B\right| \geq C|B|$, where $C>0$ is independent of $B_{0}$ and $B$. Plugging this into (5.14), we arrive at estimate (5.4). Next, we can repeat the arguments of the preceding proof (including the application of Lemma 4.2) to complete the proof of Theorem 2.4.

\section{REFERENCES}

[1] V. G. Maz'ya, Sobolev spaces, Leningrad. Gos. Univ., Leningrad, 1985; English transl., SpringerVerlag, Berlin, 1985. MR0807364 (87g:46055)] MR0817985 (87g:46056)

[2] J. Moser, On Harnack's theorem for elliptic differential equations, Comm. Pure Appl. Math. 14 (1961), 577-591. MR0159138(28:2356)

[3] B. Franchi and E. Lanconelli, Hölder regularity theorem for a class of linear nonuniformly elliptic operators with measurable coefficients, Ann. Scuola Norm. Sup. Pisa Cl. Sci. (4) 10 (1983), 523-541. MR0753153 (85k:35094)

[4] F. Chiarenza and R. Serapioni, A Harnack inequality for degenerate parabolic equations, Comm. Partial Differential Equations 9 (1984), 719-749. MR0748366 (86c:35082)

[5] E. T. Sawyer and R. L. Wheeden, Weighted inequalities for fractional integrals on Euclidean and homogeneous spaces, Amer. J. Math. 114 (1992), 813-874. MR1175693 (94i:42024)

[6] P. Pansu, Métriques de Carnot-Carathéodory et quasiisométries des espaces symétriques de rang un, Ann. of Math. (2) 129 (1989), 1-60. MR0979599 (90e:53058)

[7] M. Gromov, Carnot-Carathéodory spaces seen from within, Sub-Riemannian Geometry, Progr. Math., vol. 144, Birkhäuser, Basel, 1996, pp. 79-323. MR.1421823(2000f:53034)

[8] B. Franchi, Weighted Sobolev-Poincaré inequalities and pointwise estimates for a class of degenerate elliptic equations, Trans. Amer. Math. Soc. 327 (1991), 125-158. MR1040042 (91m:35095)

[9] B. Franchi, C. E. Guttiérez, and R. L. Wheeden, Weighted Sobolev-Poincaré inequalities for Grushin type operators, Comm. Partial Differential Equations 19 (1994), 523-604. MR1265808 (96h:26019)

[10] L. Hörmander, Hypoelliptic second order differential equations, Acta Math. 119 (1967), 147-171. MR 0222474(36:5526)

[11] L. Capogna, D. Danielli, and N. Garofalo, An embedding theorem and the Harnack inequality for nonlinear subelliptic equations, Comm. Partial Differential Equations 18 (1993), 1765-1794. MR.1239930 (94j:35038)

[12] $\ldots$, The geometric Sobolev embedding for vector fields and the isoperimetric inequality, Comm. Anal. Geom. 2 (1994), 203-215. MR1312686 (96d:46032)

[13] D. Danielli, Regularity at the boundary for solutions of nonlinear subelliptic equations, Indiana Univ. Math. J. 44 (1995), 269-286. MR1336442 (97b:35028)

[14] M. de Guzmán, Differentiation of integrals in $R^{n}$, Lecture Notes in Math., vol. 481, Springer-Verlag, Berlin-New York, 1975. MR 0457661 (56:15866)

[15] C. Fefferman and D. H. Phong, Subelliptic eigenvalue problems, Conference on Harmonic Analysis in Honor of Antoni Zygmund, Vols. I, II (Chicago, Ill., 1981), Wadsworth, Belmont, CA, 1983, pp. 590-606. MR0730094 (86c:35112)

[16] P. Hajlasz and P. Strzelecki, Subelliptic p-harmonic maps into spheres and the ghost of Hardy spaces, Max-Planck-Inst. Mat. Naturwiss., Leipzig, Preprint no. 36, 1997, pp. 1-22. Math. Ann. 312 (1998), 341-362. MR 1671796 (2000b:35033)

[17] A. Nagel, E. M. Stein, and S. Wainger, Balls and metrics defined by vector fields. I. Basic properties, Acta Math. 155 (1985), 103-147. MR0793239 (86k:46049)

[18] N. Garofalo and D. Nhieu, Isoperimetric and Sobolev inequalities for Carnot-Carathéodory spaces and the existence of minimal surfaces, Comm. Pure Appl. Math. 49 (1996), 1081-1144. MR1404326 (97i:58032)

[19] G. B. Folland, Subelliptic estimates and function spaces on nilpotent Lie groups, Ark. Mat. 13 (1975), 161-207. MR0494315 (58:13215)

[20] D. Jerison, The Poincaré inequality for vector fields satisfying Hörmander's condition, Duke Math. J. 53 (1986), 503-523. MR0850547 (87i:35027)

[21] G. Lu, The sharp Poincaré inequality for free vector fields: An endpoint result, Preprint, 1992; Rev. Math. Iberoamericana 10 (1994), 453-466. MR.1286482 (96g:26023) 
[22] B. Franchi, G. Lu, and R. L. Wheeden, Representation formulas and weighted Poincaré inequalities for Hörmander vector fields, Ann. Inst. Fourier (Grenoble) 45 (1995), 577-604. MR.1343563 (96i:46037)

[23] L. Saloff-Coste, A note on Poincaré, Sobolev, and Harnack inequalities, Internat. Math. Res. Notices 1992, no. 2, 27-38. MR.1150597 (93d:58158)

[24] D. Danielli, Formules de représentation et théorèmes d'inclusion pour des opérateurs sous elliptiques, C. R. Acad. Sci. Paris Sér. I Math. 314 (1992), 987-990. MR.1168522 (93e:35020)

[25] C. Pérez, Two weighted norm inequalities for Riesz potentials and uniform $L^{p}$-weighted Sobolev inequalities, Indiana Univ. Math. J. 39 (1990), 31-44. MR1052009 (92a:42024)

[26] H. Federer, Geometric measure theory, Grundlehren Math. Wiss., Bd. 153, Springer-Verlag New York, Inc., New York, 1969. MR0257325 (41:1976)

[27] W. Fleming and R. Rishel, An integral formula for the total gradient variation, Arch. Math. 11 (1960), 218-222. MR0114892(22:5710)

Institute of Mathematics and Mechanics, National Academy of Sciences, Azerbaidzhan, And Dichle University, Diyarbakir, Turkey

E-mail address: farman-m@mail.ru

Institute of Mathematics and Mechanics, National Academy of Sciences, Azerbaidzhan

E-mail address: rabilamanov@hotmail.com

Received 14/JUN/2006

Translated by A. PLOTKIN 